\title{
Fabrication of metallic patterns by microstencil lithography on polymer surfaces suitable as microelectrodes in integrated microfluidic systems
}

\author{
Nao Takano ${ }^{1}$, Lianne M Doeswijk ${ }^{1}$, Marc A F van den Boogaart ${ }^{1}$, \\ Janko Auerswald ${ }^{2}$, Helmut F Knapp ${ }^{2}$, Olivier Dubochet ${ }^{3}$, \\ Thomas Hessler $^{3}$ and Jürgen Brugger ${ }^{1}$ \\ ${ }^{1}$ Microsystems Laboratory, École Polytechnique Fédérale de Lausanne (EPFL), \\ 1015 Lausanne, Switzerland \\ ${ }^{2}$ Centre Suisse d'Electronique et de Microtechnique (CSEM SA), Division Alpnach, \\ 6055 Alpnach, Switzerland \\ ${ }^{3}$ Leister Process Technologies, Axetris Microsystems Division, 6056 Kägiswil, Switzerland \\ E-mail: nao.takano@epfl.ch and juergen.brugger@epfl.ch
}

Received 30 March 2006, in final form 5 June 2006

Published 3 July 2006

Online at stacks.iop.org/JMM/16/1606

\begin{abstract}
Microstencil lithography, i.e. local deposition of micrometer scale patterns through small shadow masks, is a promising method for metal micropattern definition on polymer substrates that cannot be structured using organic-solvent-based photoresist technology. We propose to apply microstencil lithography to fabricate microelectrodes on flat and 3D polymer substrates, such as PMMA or SU-8, which form parts of microfluidic systems with integrated microelectrodes. Microstencil lithography is accompanied by two main issues when considered for application as a low-cost, reproducible alternative to standard photolithography on polymer substrates. In this paper we assess in detail (i) the reduction of aperture size (clogging) after several metal evaporation steps and corresponding change of deposited pattern size and (ii) loss in the resolution (blurring) of the deposited microstructures when there is a several micrometers large gap between the stencil membrane and the substrate. The clogging of stencil apertures induced by titanium and copper evaporation was checked after each evaporation step, and it was determined that approximately $50 \%$ of the thickness of the evaporated metals was deposited on the side walls of the stencil apertures. The influence of a gap on the deposited structures was analyzed by using $18 \mu \mathrm{m}$ thick SU-8 spacers placed between the microstencil and the substrate. The presence of an $18 \mu \mathrm{m}$ gap made the deposited structures notably blurred. The blurring mechanism of deposited structures is discussed based on a simplified geometrical model. The results obtained in this paper allow assessing the feasibility of using stencil-based lithography for unconventional surface patterning, which shows the limits of the proposed method, but also provides a guideline on a possible implementation for combined polymer-electrode microsystems, where standard photoresist technology fails.
\end{abstract}

(Some figures in this article are in colour only in the electronic version) 


\section{Introduction}

Microfluidic systems with incorporated microelectrodes are adopted in a variety of applications in life sciences, such as impedance spectroscopy cell analysis [1, 2], capillary electrophoresis [3, 4], dielectrophoresis [5-8], or isoelectric focusing devices [9], microsystems for electrostatic droplet separation, mixing and steering [10-12], micropumps [13] and micro-electrode recording arrays for the study of cellular activity $[14,15]$. Most of these microelectrofluidic devices are fabricated based on glass, silicon and silicon-related materials' micromachining processes, which are expensive for the production of disposable or semi-disposable sensing devices. The lack of a low-cost production method for such devices is one of the major reasons today, why the real breakthrough of disposable microfluidic systems with integrated microelectrodes in the life sciences and especially in the field of diagnostics has not been achieved.

Plastics and other polymer materials are advantageous to be used as substrates of microfluidic systems in order to realize a low-cost production process of devices, because these materials can be easily structured by e.g. hotembossing, injection molding or thermal forming with excellent reproducibility. Using polymers would also help facilitate some processes, such as bonding chips and drilling holes, compared to processing glass substrates that are commonly used for microfluidic devices. However, the lithographical process of microelectrode fabrication on polymer substrates is one of the critical issues, because many of these materials cannot withstand organic solvents which are unavoidable during conventional photolithography processes. To overcome this issue, there are two main ways: (a) use of a photoresist solvable in a water-based solution and (b) use of new emerging lithography processes instead of conventional photolithography processes. A lift-off process to make platinum $(\mathrm{Pt})$ electrodes on a polymethylmetacrylate (PMMA) substrate by using a novolac-based photoresist containing no solvents that dissolve PMMA was performed by Grass $e t$ al, in which the novolac-based photoresist could be developed by an aqueous-based solution [16]. Although the resolution of this photoresist is not clearly mentioned in this paper (the smallest parts of the electrodes were $50 \mu \mathrm{m}$ in width), this process has a high potential for the realization of metal electrodes on polymer substrates.

Selective deposition or pattern transfer onto polymer substrates can also be accomplished by depositing metal through miniaturized stencils (or shadow masks) [17-23], the so-called micro/nano stencil lithography. Since microstencil lithography is a resistless, single-step direct vacuum patterning method, it enables us to simplify the device fabrication process. Moreover, the method is capable of making nanoor microstructures on non-conventional substrates, such as polymers or various substrates covered with self-assembled monolayers (SAMs), without damaging and contaminating the substrates by organic solvents/compounds that are contained in most photoresists and that are necessary for developing patterns [24-26]. In this study, we investigated the application of microstencil lithography to microelectrofluidic device fabrication and focused on a quantitative analysis of two specific issues accompanying microstencil lithography: clogging and blurring.
One of the main limitations of microstencil lithography is that the method is associated with gradual aperture clogging (figure 1(a)). To suppress the clogging phenomenon, coating stencils with SAMs is highly helpful, by which the useful lifetime of stencils can be extended [21, 27]. In this study, however, we traced the clogging of microapertures without SAM coating by using titanium (Ti) and copper $(\mathrm{Cu})$ as deposited metals by an observation of the stencil apertures and deposited microstructures after each evaporation step.

The other drawback of stencil lithography is related to the presence of a gap between the stencil and the substrate (figure 1(b)). For instance, microfluidic channels can be structured by hot embossing, thermoforming or chip bonding either before or after the microstencil evaporation. However, in the former case, i.e., structuring microfluidic channels followed by microelectrode fabrication by microstencil lithography, a gap corresponding to the channel depth will be present between the substrate and the microstencil especially if electrodes are required both on the channel top and at the bottom, which causes blurring of deposited microstructures. Although microstructure fabrication on 3Dstructured substrates can be accomplished with reduced risk of structure blurring due to gap by employing a 3D shadow mask [28], this technique requires complex processing and is costly. Therefore, we examined the influence of the presence of gap on the deposited microstructures by using SU-8 structures as spacers and compared the experimental results with a simple geometrical model to assess the feasibility of microstencil lithography using planar microstencils.

Microstencil lithography can also suffer from membrane deformation due to the deposition-induced stresses (figure 1(c)). Design variation can be limited by this membrane deformation issue, which is critical particularly when complex structures need to be realized with stencil lithography, as often encountered by integrated microelectrode configurations. Recently, however, some smart solutions have been proposed and demonstrated in order to reduce the membrane deformation by local mechanical reinforcement. Further details on the solutions of this issue are discussed elsewhere [29, 30].

\section{Experimental details}

The microstencils used for these experiments were fabricated in a $100 \mathrm{~mm}$ wafer by silicon micromachining processes which are described in detail elsewhere [22, 23]. As illustrated in figure 1, a microstencil consists of a low-stress silicon nitride $(\mathrm{SiN})$ membrane $(500 \mathrm{~nm}$ thick) with a variety of microapertures and a silicon support. The wafer-scale microstencil was cut into chips $\left(6 \times 6 \mathrm{~mm}^{2}, 18 \times 18 \mathrm{~mm}^{2}\right)$ which contain 4 and 49 stencil membranes, respectively, that are $1 \times 1 \mathrm{~mm}^{2}$ in size with various designs. In this study, the microstencil chips were fixed onto substrates by using polyimide tape during the evaporation process.

For the metal deposition process, electron beam evaporation (Alcatel, EVA 600) was employed since the metal evaporation is a rather directional physical vapor deposition method, so that well-defined structures are obtained with microstencil lithography. We chose titanium as a material for the microstructures since it shows good adhesion and 




Figure 1. Schematic representation of the microstencil lithography technique and its limitations $(a)-(c)$.

biocompatibility. In each experiment, the thickness of deposited Ti was $200 \mathrm{~nm}$. Copper was also used for the study of aperture clogging.

In order to create a fixed gap between the microstencil and the substrate for the purpose of simulating the presence of microfluidic channels, $18 \mu \mathrm{m}$ thick SU-8 (MicroChem Corp., SU-8 2015) structures were fabricated on $\mathrm{SiO}_{2}(200 \mathrm{~nm}) / \mathrm{Si}$ substrates by conventional photolithography processes. The $\mathrm{SiO}_{2}$ layer was formed by a thermal oxidation process under an $\mathrm{H}_{2} \mathrm{O}$ vapor atmosphere. The microstencils were either placed on the SU-8 structures or directly on the $\mathrm{SiO}_{2}$ surface to examine the influence of the gap on the deposited structures. For the characterization of microstencils and fabricated microstructures, a scanning electron microscope (SEM; Leo Electron Microscopy Ltd., LEO 1550), an atomic force microscope (AFM; Nanosurf AG, easyScan DFM) and an optical microscope (Nikon Corp., Optiphot 200) were employed.

\section{Results and discussion}

\subsection{Clogging of microstencil apertures and its influence on deposited structures}

One of the possible advantages of microstencil lithography is that the microstencils can be used repeatedly, which allows for reducing the production costs of devices. Since each device has its own requirements for electrode structures, it is important to analyze how the microstencil apertures are altered after multiple metal depositions, and how the size of corresponding microstructures deposited through the microstencil varies in accordance with the clogging.

In figures 2 and $3(a),(c),(e)$, the variation of microstencil aperture $W$ as a function of Ti evaporation $t_{\text {dep }}$ is shown. The aperture, initially $4.8 \mu \mathrm{m}$ wide $\left(W_{0}\right)$, reduced linearly according to the increase in the total thickness of evaporated Ti (200 nm/evaporation). The thickness of deposited Ti on the side wall of the aperture was $0.1 \mu \mathrm{m}$ per evaporation, which corresponds to $50 \%$ of the evaporated Ti thickness $t_{\text {dep }}$. Similar values were observed when $\mathrm{Cu}$ was used as a deposited material (represented by filled dots in figure 2).

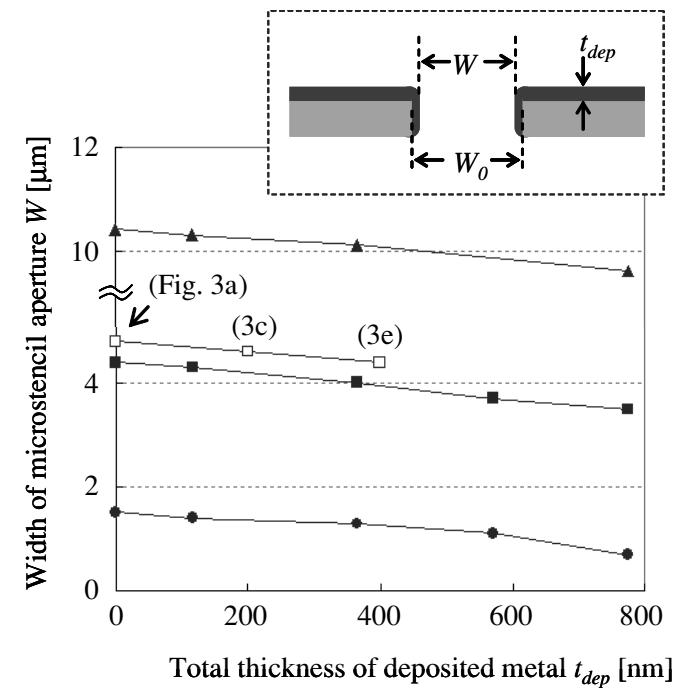

Figure 2. Clogging of the microstencil apertures as a function of deposited $\mathrm{Ti}$ (open dots) and $\mathrm{Cu}$ (filled dots) film thicknesses by e-beam evaporation. A schematic cross-sectional illustration of an aperture of stencil membrane is shown in the inset.

The deposited $\mathrm{Ti}$ at the aperture edge is better visible in figure $4(a)$, which shows the same part of the microstencil as figures $3(a),(c)$ and $(e)$ but observed from the opposite side of the stencil membrane, i.e., observed from the side having faced the substrate during the evaporation as illustrated above the SEM images. When figure 4(a) is compared with figure 3(a), it suggests that evaporated Ti has diffused to the other side of the membrane around the aperture. Figure $4(b)$ shows enlargements of a part of figure $4(a)$ indicated by the dotted rectangle, in which we can clearly see the progress of clogging.

Figures $3(b)$ and $(d)$ show the Ti structures deposited through the microstencil that is shown in figures $3(a),(c),(e)$ and 4 . The width of the deposited structures reduced according to the reduction of the aperture size of the microstencil. Since the shape of this aperture is a long rectangle of about $5 \mu \mathrm{m}$ width, the SiN membrane of the microstencil was barely influenced by the residual stress of the deposited Ti layer, 


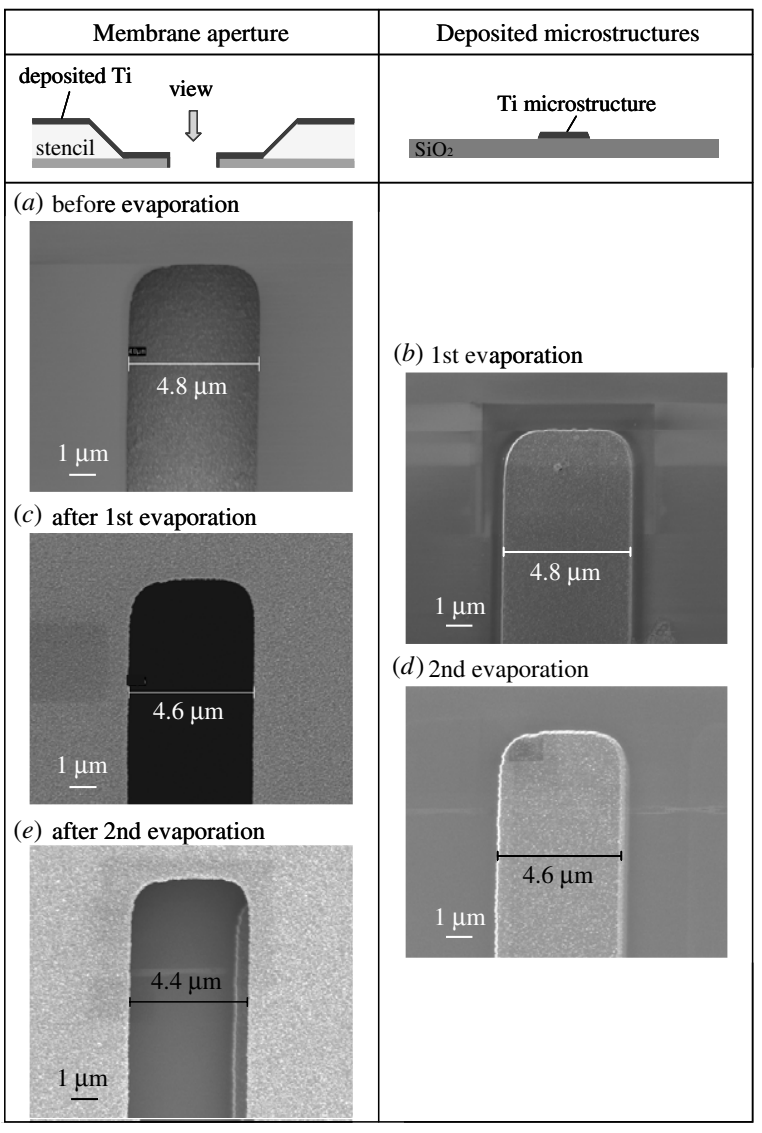

Figure 3. SEM images of a part of the membrane aperture $(a),(c)$, $(e)$ observed from the side having faced the incoming Ti flux during the evaporation (see figure 1), and of Ti structures deposited through the microstencil $(b),(d)$ on $\mathrm{SiO}_{2}$ surfaces. Each image of membrane aperture corresponds to the points indicated in the graph in figure 2; (a) membrane aperture before evaporation of $\mathrm{Ti},(b)$ Ti structure obtained at first evaporation, $(c)$ membrane aperture after first evaporation of $\mathrm{Ti},(d) \mathrm{Ti}$ structure obtained at second evaporation and $(e)$ membrane aperture after second evaporation of $\mathrm{Ti}(200 \mathrm{~nm} /$ evaporation).

which resulted in a faithful pattern transfer even after multiple usages.

The above-mentioned results suggest that the microstencils can be used several times even without a cleaning treatment when the device specifications allow some micrometer tolerance of the electrode size, e.g. for a microstencil with $20 \mu \mathrm{m}$ wide apertures, ten times deposition cycles of $200 \mathrm{~nm}$ thick microelectrodes without cleaning would result in $1 \%$ error that is acceptable for several applications. In order to apply microstencil lithography to a large-scale production, however, cleaning treatments to recycle clogged stencils are inevitable.

\subsection{Influence of the gap between microstencil and substrate on deposited structures}

To demonstrate the ability of microstructure fabrication by stencil lithography on polymer substrates, Ti microstructure fabrication was performed by using microstencils on a PMMA substrate (figure 5(a)) which is a good dielectric, biocompatible, transparent material and can be easily
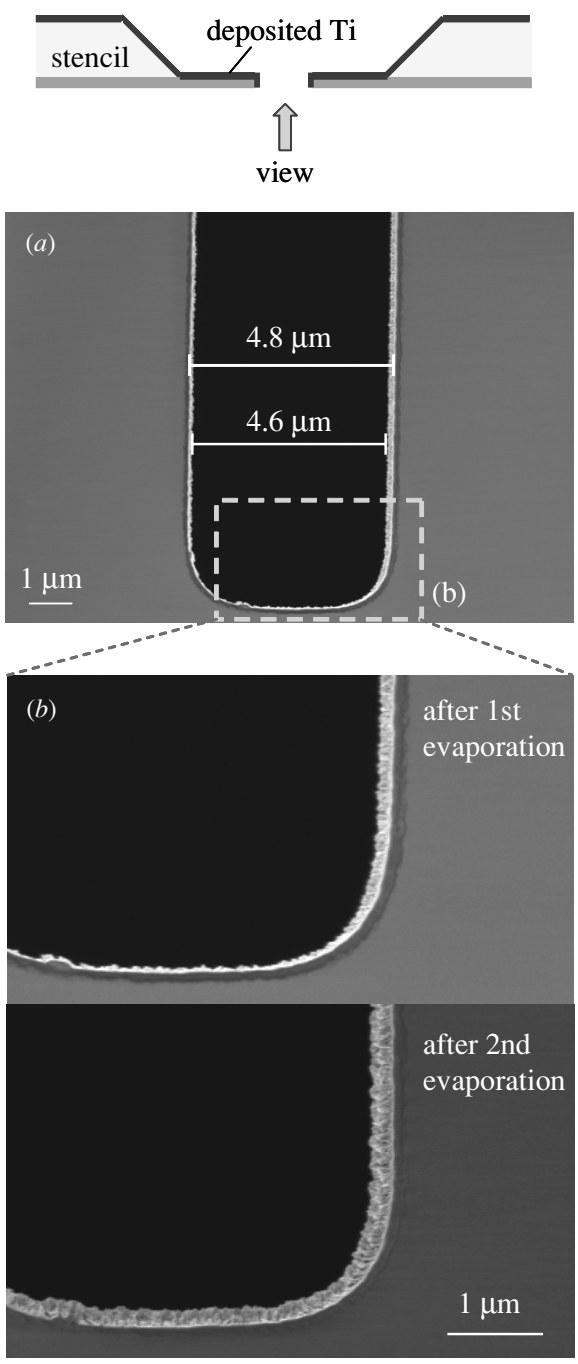

Figure 4. SEM images of the microstencil observed from the side having faced the substrate during the evaporation (opposite side of stencil shown in figure 3); (a) after first evaporation, (b) enlargement of a part of figure $4(a)$ indicated by the dotted rectangle, after first and second evaporations ( $200 \mathrm{~nm} /$ evaporation) of Ti. Progress of the clogging at the edge of aperture can be clearly observed.

processed with standard fabrication techniques. The upper image of figure $5(a)$ is the optical micrograph of $\mathrm{Ti}$ microstructures fabricated on a PMMA substrate. The AFM image shown below the optical image corresponds to a part of the 'P'-shaped structure of the largest EPFL logo (our test pattern) indicated by the dotted circle shown in the optical micrograph. Microstructure fabrication was also performed on an SU-8 substrate as shown in figure $5(b)$. Both results in figure 5 confirm that the structures obtained by microstencil lithography have well-defined edges when deposited on polymer substrates.

Although figure 5 demonstrates good pattern transfer with microstencil lithography on a flat polymer substrate, we need to consider the complete device fabrication. To fabricate microfluidic devices with integrated microelectrodes onto polymer substrates by employing microstencil lithography, there are mainly two possible procedures: (i) microelectrode fabrication by microstencil lithography on a pre-structured 


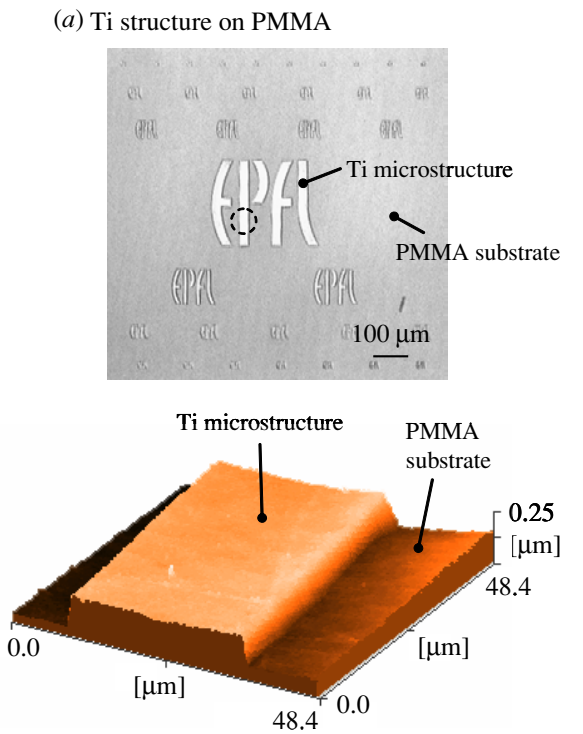

(b) Ti structure on SU-8

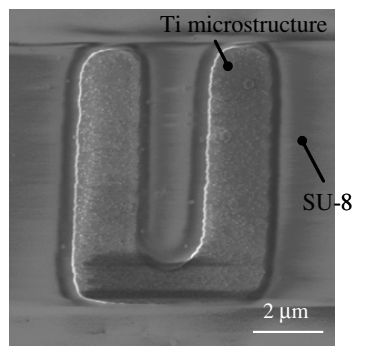

Figure 5. Ti microstructures deposited through a microstencil on polymer substrates: (a) optical micrograph of the Ti structure on PMMA (top) and 3D-view AFM image which corresponds to a part of the optical micrograph indicated by the dotted circle and (b) SEM image of the Ti structure on SU-8.

polymer substrate, or (ii) microelectrode fabrication by microstencil lithography on a flat polymer substrate followed by microfluidic channel fabrication steps. If microstencil lithography is performed on a 3D-structured polymer substrate (case (i)), the fabrication process of a device would be simplified. In this case, however, the influence of a gap between the microstencil and the lower part of a substrate has to be inspected, since the presence of gap often induces blurring of deposited microstructures.

Assuming a typical microfluidic channel for dielectrophoresis or capacitive flow cytometry with a depth of about $18 \mu \mathrm{m}$, the influence of the gap was examined by using $\mathrm{SU}-8$ spacers fabricated on an $\mathrm{SiO}_{2} / \mathrm{Si}$ substrate. Two microstencils that contain precisely the same designs are placed on the same wafer about $2.5 \mathrm{~cm}$ apart, one on the top of the SU-8 spacers and the other directly on $\mathrm{SiO}_{2}$. In order to avoid any material-dependent influence, such as surface diffusion of $\mathrm{Ti}$ as well as charging effect, we deposited $\mathrm{Ti}$ microstructures on $\mathrm{SiO}_{2}$ surfaces in both cases of with and without gap. The Ti microstructure in figure 6(a) was deposited through the microstencil directly placed on the $\mathrm{SiO}_{2}$ surface, while there was an $18 \mu \mathrm{m}$ gap between the microstencil and the $\mathrm{SiO}_{2}$ surface where the structure in figure $6(b)$ was deposited (see the schematic representations above the SEM images). The SEM image in figure $6(b)$ shows that the presence of an $18 \mu \mathrm{m}$ gap results in a blurred structure. The difference of structure edge definition is more visible in the AFM images and their cross-sectional line views, which are shown in figures $6(c)-(f)$. Hence, we conclude that it is possible to fabricate microstructures with the presence of a gap between the stencil and the substrate, but the presence of a gap diminishes the edge definition of deposited microstructures and their spatial resolution.

Figure 7 shows simple models that explain the blurring of deposited structures when there is a gap between a microstencil and a substrate. Since the mean free path of evaporated metal particles is much longer than the distance between the metal source and the substrate under our electron beam evaporation condition ( $\leqslant 10^{-6}$ mbar), we can assume that the evaporated metal proceeds in a straight line of sight fashion from the source to the substrate as illustrated in the geometrical models in figure $7[17,18]$. There are mainly two situations of the membrane aperture toward the metal source which have been considered: (i) the membrane aperture situated right below the metal source, i.e., between $A$ and $A^{\prime}$, which corresponds to the center part of a wafer (figure $7(a)$ ), and (ii) the membrane aperture is outside the perpendicular projection of the source on the membrane, i.e., the distance between $A_{0}$ and the membrane aperture, $d$, is larger than the radius of metal source $R$ (figure $7(b)$ ). For simplicity, we define the structure blurring $B$ as $w-2 r$, where $w$ is the deposited structure width and $2 r$ is the aperture width. In the situation of figure 7(a), i.e., the membrane aperture is within the perpendicular projection of the metal source, the structure blurring $B$ can be calculated as $B \approx 2(R+r) G / D$ under the condition of $D \gg t$, where $G$ is the gap between the microstencil membrane and the substrate, $D$ is the distance between the metal source and the membrane and $t$ is the thickness of the stencil membrane. Since the values of $R\left(7.5 \times 10^{-3} \mathrm{~m}\right), D\left(5.0 \times 10^{-1} \mathrm{~m}\right)$ and $t(5.0 \times$ $\left.10^{-7} \mathrm{~m}\right)$ are fixed in our experiments, and $r$ is negligibly small compared to the other parameters such as $R$ and $D$, the blurring of microstructures can principally be attributed to the value of $G$. The situation of figure $7(b)$ is a little more complex since the distance between the perpendicular projection of the center of metal source $\left(A_{0}\right)$ and the membrane aperture, $d$, needs to be considered. In this case the structure blurring $B$ can be calculated as $B \approx\{(R+d+2 r) G-(d-R)(G+t)\} / D$, also under the condition of $D \gg t$, and the main factors influencing the structure blurring are the values of $G$ and $d$. Assuming that the stencil membrane is infinitely thin, the structure blurring $B$ is independent of the distance $d$, as it is obvious from the equation. However, as the distance $d$ increases, the cross section of the deposited structure changes as illustrated in figure 8 . When the membrane aperture is situated right under the metal source, which corresponds to the situation of figure 7(a), the deposited structure has almost symmetric cross section (figure 8(i)), while the blurred region becomes larger on one side (figure 8(ii)) under the situation of figure $7(b)$. Additionally, the structure blurring is more influenced when the membrane thickness becomes larger due to the shadow effect caused by the thickness of the membrane. All of these phenomena need to be taken into consideration when designing a device configuration including overall production processes. 

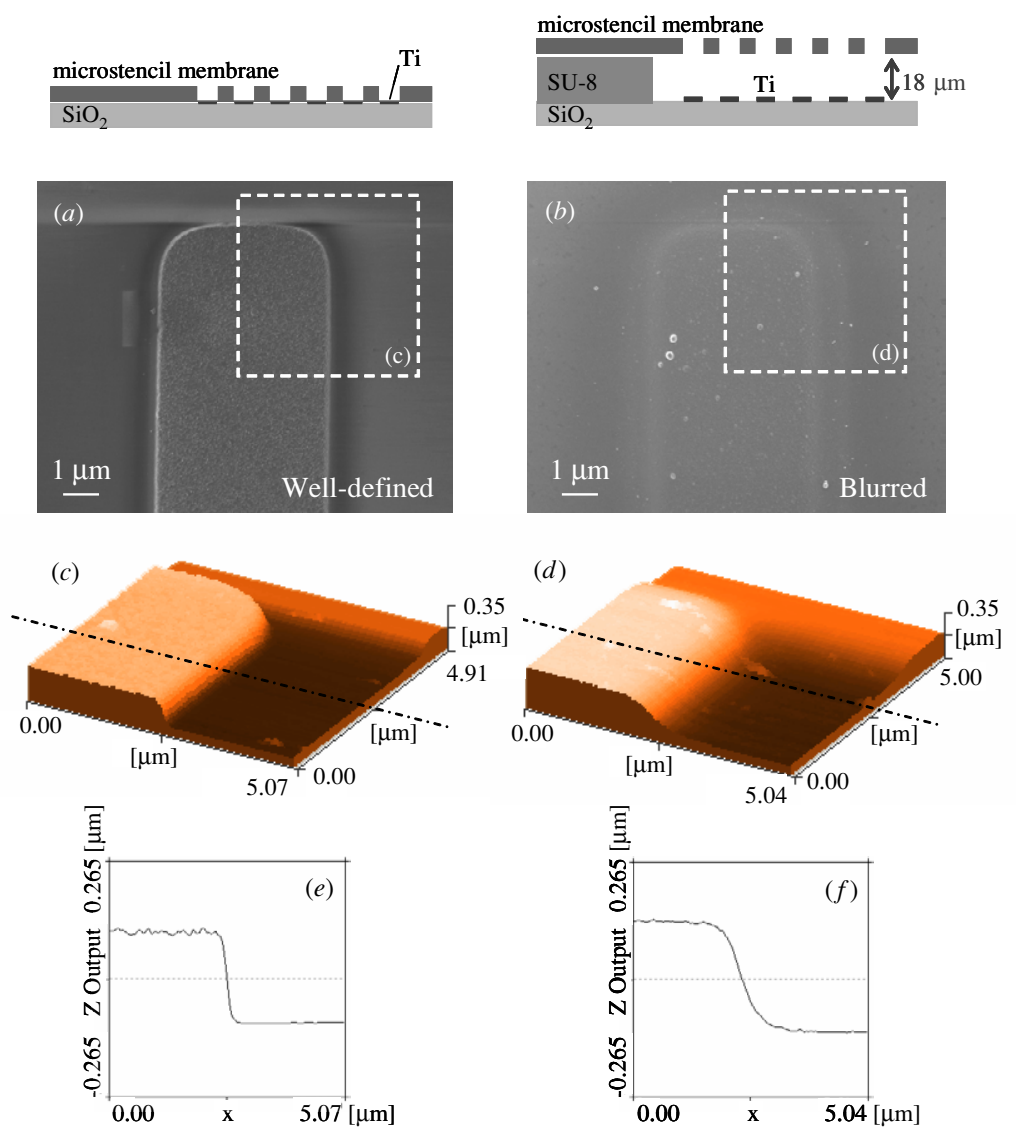

Figure 6. Comparison of Ti microstructures deposited on the same material surface $\left(\mathrm{SiO}_{2}\right)$ without and with a gap: $(a)$ and $(b)$ SEM images, shown with schematic cross-sectional illustration of the sample setup during the evaporation, without SU-8 spacer and with $18 \mu \mathrm{m}$ thick SU-8 spacer, respectively, $(c)$ and $(d)$ AFM images of a part of figures 6(a) and $(b)$ (indicated by dotted square), respectively, $(e)$ and $(f)$ cross-sectional line views of the AFM images in $(c)$ and $(d)$, indicated with broken lines.
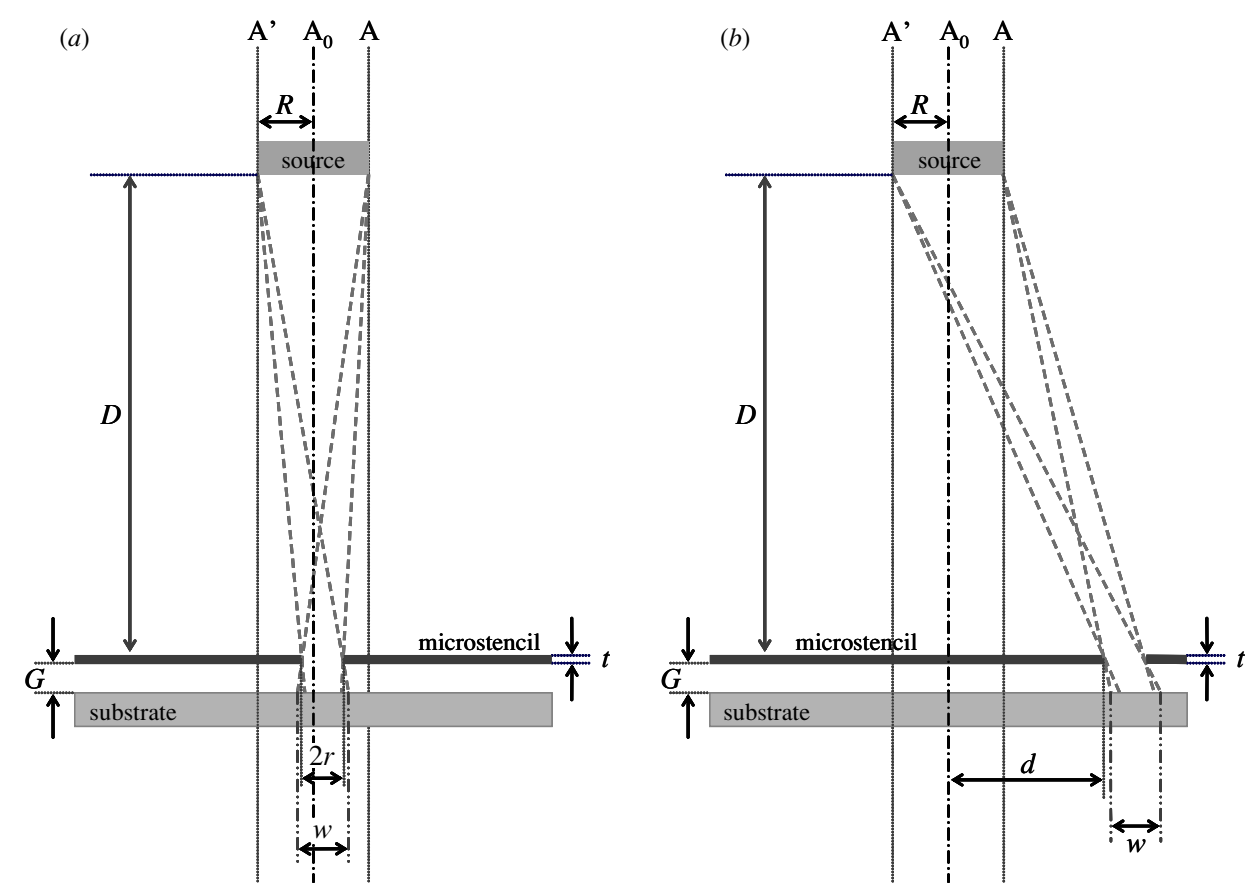

Figure 7. Simplified models explaining the blurring mechanism of deposited structures by having a gap between a microstencil and a substrate: $(a)$ a membrane aperture is placed between $A$ and $A^{\prime}$, and $(b)$ a membrane aperture is outside of the perpendicular projection of the metal source $(d \geqslant R)$. 


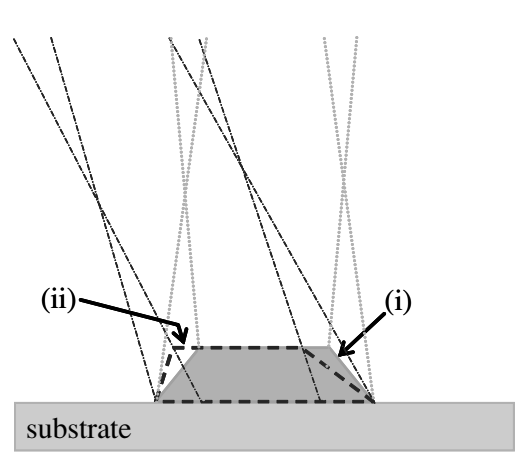

Figure 8. Schematic cross-sectional illustration of the microstructures deposited through a microstencil under the conditions of figures 7(a) (i): solid and (b) (ii): dotted line.

In the following, we compare the experimental results with the calculated structure width $w$ under the simplest situation, the case of figure 7(a), based on the approximation that the influence of the membrane thickness $t$ on the structure blurring $B$ was not significant when the Ti microstructure in figure $6(b)$ was deposited. For the stencil aperture (width $4.5 \mu \mathrm{m}$ ) used to make the structures in figure $6(b)$, the expected width of the deposited structure $w$ (calculated as $2 r+B$ ) over an $18 \mu \mathrm{m}$ gap should be $5.0 \mu \mathrm{m}$ according to this model. As determined from the SEM image in figure $6(b)$, the width of the deposited structure over the $18 \mu \mathrm{m}$ gap is $6.0 \mu \mathrm{m}$, which is larger than the calculated value $(5.0 \mu \mathrm{m})$. Although the model provides a first indication of the size of the deposited structures, we can conclude that some other effects, such as surface diffusive motion of evaporated $\mathrm{Ti}$, should be also taken into account. Concerned with the influence of the surface diffusion of evaporated metal on blurring, we observed clear difference between $\mathrm{Cu}$ and $\mathrm{Al}$ microstructures deposited on Si through the microstencils. The blurred regions of $\mathrm{Cu}$ microstructures were much larger than that of $\mathrm{Al}$ [31]. Further details are under investigation and will be published elsewhere. We conclude that performing microstencil lithography on a flat surface is most preferable in order to obtain well-defined microelectrode structures required for the microfluidic systems. With all effects included, the change of dimension of the micropattern defined through a $4.5 \mu \mathrm{m}$ stencil aperture over a gap of $18 \mu \mathrm{m}$ (i.e., 3-4 times larger than the aperture size) resulted in a pattern width of $6.0 \mu \mathrm{m}$.

\section{Conclusions}

The influence of possible drawbacks accompanying microstencil lithography on the fabrication process of polymer microfluidic devices incorporating microelectrodes was investigated. The variation in the size of microstencil apertures after several evaporation steps was checked step by step, which revealed that about $50 \%$ of the intended thickness of the evaporated metals deposited on the sidewalls of the apertures. The size of the deposited $\mathrm{Ti}$ structures was in a good agreement with the aperture size. The gradual size reduction by the clogging offers the possibility of repetitive usage of microstencils depending on device requirements, which can be an advantage for the large-scale production of devices. The resolution of deposited microstructures with/without a gap between a stencil and a substrate was also examined in order to optimize a procedure to make microfluidic channels on polymer substrates with integrated microelectrodes. The experimental results and the geometrical stencil evaporation model showed that the presence of gap was the major cause of blurring. It is also suggested that the surface diffusion of evaporated metal is an important factor which influences the structure blurriness. We conclude that performing microstencil lithography on a flat surface followed by microfluidic channel fabrication processes is most preferable in order to obtain well-defined microelectrode structures required for the microfluidic systems. In addition to the gap control, it is also practicable to reduce blurring by increasing the distance between the microstencil membrane and the evaporation source $D$ as is clearly suggested from the geometrical model, which will be necessary to be taken into consideration when an overall device production procedure is designed. The results presented here allow assessing the feasibility, and limits, of integrated microelectrode fabrication on polymer substrates having 3D surface features useful for integrated microfluidic system fabrication by means of microstencil lithography.

\section{Acknowledgments}

The authors are grateful to the EPFL Center of Micro- and Nano-Technology (CMI) for their great help. This work was partially financed by the Swiss Commission for Technology and Innovation (CTI) under the project CTI 6640.2 NMS$\mathrm{NM}$, and the Swiss Federal Office for Science and Education (OFES) in the framework of the EC-funded FP6 project $\mathrm{NaPa}$ (NMP4-CT-2003-500120).

\section{References}

[1] Ayliffe H E, Frazier A B and Rabbitt R D 1999 Electric impedance spectroscopy using microchannels with integrated metal electrodes J. Microelectromech. Syst. 8 50-7

[2] Gawad S, Schild L and Renaud Ph 2001 Micromachined impedance spectroscopy flow cytometer for cell analysis and particle sizing Lab Chip 1 76-82

[3] Guijt R M, Baltussen E, van der Steen G, Schasfoort R B M, Schlautmann S, Billiet H A H, Frank J, van Dedem G W K and van den Berg A 2001 New approaches for fabrication of microfluidic capillary electrophoresis devices with on-chip conductivity detection Electrophoresis 22 235-41

[4] Baldwin R P et al 2002 Fully integrated on-chip electrochemical detection for capillary electrophoresis in a microfabricated device Anal. Chem. 74 3690-7

[5] Docoslis A, Kalogerakis N, Behie L A and Kaler K V I S 1997 A novel dielectrophoresis-based device for the selective retention of viable cells in cell culture media Biotechnol. Bioeng. 54 239-50

[6] Xu C, Wang Y, Cao M and Lu Z 1999 Dielectrophoresis of human red cells in microchips Electrophoresis 20 1829-31

[7] Green N G, Ramos A and Morgan H 2000 Ac electrokinetics: a survey of sub-micrometre particle dynamics J. Phys. D: Appl. Phys. 33 632-41

[8] Auerswald J and Knapp H F 2003 Quantitative assessment of dielectrophoresis as a micro fluidic retention and separation technique for beads and human blood erythrocytes Microelectron. Eng. 67-8 879-86 
[9] Voldman J, Gray M L, Toner M and Schmidt M A 2002 A microfabrication-based dynamic array cytometer Anal. Chem. 74 3984-90

[10] Latorre L, Kim J, Lee J, de Guzman P P, Lee H J, Nouet P and Kim C J 2002 Electrostatic actuation of microscale liquid-metal droplets J. Microelectromech. Syst. 11 302-8

[11] Cho S K, Moon H and Kim C J 2003 Creating, transporting, cutting, and merging liquid droplets by electrowettingbased actuation for digital microfluidic circuits J. Microelectromech. Syst. 12 70-80

[12] Fan S K, Hashi C and Kim C J 2003 Manipulation of multiple droplets on $\mathrm{N} \times \mathrm{M}$ grid by cross-reference EWOD driving scheme and pressure-contact packaging IEEE Conf. on MEMS (Kyoto, Japan)

[13] Yun K S, Cho I J, Bu J U, Kim C J and Yoon E 2002 A surface-tension driven micropump for low-voltage and low-power operations J. Microelectromech. Syst. 11 454-61

[14] Moldovan C, Ilian V, Constantin G, Iosub R, Modreanu M, Dinoiu I, Firtat B and Voitincu C 2002 Micromachining of a silicon multichannel microprobe for neural electrical activity recording Sensors Actuators A 99 119-24

[15] Pearce T M, Wilson J A, Oakes S G, Chiu S Y and Williams J C 2005 Integrated microelectrode array and microfluidics for temperature clamp of sensory neurons in culture Lab Chip 5 97-101

[16] Graß B, Neyer A, Jöhnck M, Siepe D, Eisenbeiß F, Weber G and Hergenröder R 2001 A new PMMA-microchip device for isotachophoresis with integrated conductivity detector Sensors Actuators B 72 249-58

[17] Burger G J, Smulders E J T, Berenschot J W,

Lammerink T S J, Fluitman J H J and Imai S 1996 High-resolution shadow-mask patterning in deep holes and its application to an electrical wafer feed-through Sensors Actuators A 54 669-73

[18] Köhler J, Albrecht M, Musil C R and Bucher E 1999 Direct growth of nanostructures by deposition through an $\mathrm{Si}_{3} \mathrm{~N}_{4}$ shadow mask Physica E 4 196-200

[19] Deshmukh M M, Ralph D C, Thomas M and Silcox J 1999 Nanofabrication using a stencil mask Appl. Phys. Lett. 75 1631-3

[20] Brugger J, Berenschot J W, Kuiper S, Nijdam W, Otter B and Elwenspoek M 2000 Resistless patterning of sub-micron structures by evaporation through nanostencils Microelectron. Eng. 53 403-5
[21] Kölbel M, Tjerkstra R W, Brugger J, van Rijn C J M, Nijdam W, Huskens J and Reinhoudt D N 2002 Shadow-mask evaporation through monolayer-modified nanostencils Nano Lett. 2 1339-43

[22] Kim G M, van den Boogaart M A F and Brugger J 2003 Fabrication and application of a full wafer size micro/nanostencil for multiple length-scale surface patterning Microelectron. Eng. 67-68 609-14

[23] van den Boogaart M A F, Kim G M, Pellens R, van den Heuvel J P and Brugger J 2004 Deep-ultravioletmicroelectromechanical systems stencils for highthroughput resistless patterning of mesoscopic structures J. Vac. Sci. Technol. B 22 3174-7

[24] Vroegindeweij F, Speets E A, Steen J A J, Brugger J and Blank D H A 2004 Appl. Phys. A 79 743-5

[25] Speets E A, Ravoo B J, Roesthuis F J G, Vroegindeweij F, Blank D H A and Reinhoudt D N 2004 Nano Lett. 4 841-4

[26] Graff M, Mohanty S K, Moss E and Frazier A B 2004 Microstencilling: a generic technology for microscale patterning of vapor deposited materials J. Microelectromech. Syst. 13 956-62

[27] Kölbel M, Tjerkstra R W, Kim G, Brugger J, van Rijn C J M, Nijdam W, Huskens J and Reinhoudt D N 2003 Self-assembled monolayer coatings on nanostencils for the reduction of materials adhesion Adv. Funct. Mater. $13219-24$

[28] Brugger J, Andreoli C, Despont M, Drechsler U, Rothuizen H and Vettiger P 1999 Self-aligned 3D shadow mask technique for patterning deeply recessed surfaces of micro-electro-mechanical systems devices Sensors Actuators A 76 329-34

[29] van den Boogaart M A F, Doeswijk L M and Brugger J 2006 Silicon supported membranes for improved large-area and high-density micro/nanostencil lithography J. Microelectromech. Syst. submitted

[30] van den Boogaart M A F, Lishchynska M, Doeswijk L M, Greer J C and Brugger J 2006 Corrugated membranes for improved pattern definition with micro/nanostencil lithography Sensors Actuators A at press, available online 19 October 2005

[31] Doeswijk L M, van den Boogaart M A F, Takano N and Brugger J 2005 Advancing towards well-controlled full-wafer nanostencil lithography Int. Conf. on Micro- and Nano Engineering 2005 (MNE 2005) (Vienna, Austria, 19-22 September 2005) 